\title{
Usage of numerical methods to solve nonlinear mixed Volterra-Fredholm integral equations and their system
}

\author{
Fawziah M. Al-saara, Kirtiwant P. Ghadle ${ }^{\mathrm{b}}$ \\ ${ }^{a}$ Department of Mathematics, Dr. Babasaheb Ambedkar Marathwada University, Aurangabad, 431004, India. \\ Department of Mathematics, Faculty of Applied Science, Amran University, Amran, Yemen. \\ ${ }^{b}$ Department of Mathematics, Dr. Babasaheb Ambedkar Marathwada University, Aurangabad-431004, India.
}

\begin{abstract}
In this paper, we apply the homotopy perturbation method (HPM), modified homotopy perturbation method (MHPM), variational iteration method (VIM), Adomian decomposition method (ADM), and modified Adomian decomposition method (MADM) to solve nonlinear mixed Volterra-Fredholm integral equations and its system. We investigate the approximate solution of this equation and its system via proposed methods. The validity and efficiency of these methods are demonstrated through various numerical examples that illustrate the efficiency, accuracy, and simplicity of the proposed methods. Moreover, the convergence and uniqueness of the solution of the suggested methods are confirmed and compared with the exact solutions.
\end{abstract}

Keywords: Nonlinear mixed Volterra-Fredholm integral equations, ADM, MADM, VIM, HPM, MHPM. 2010 MSC: 45D05, 47H30, 47Jxx, 45Gxx.

\section{Introduction}

In general, many physical problems are constituted as differential, integral and integro-differential equations. Recently a lot of numerical methods have been used by researchers to discover the analytical and approximation solution of these equations. Many mathematicians have focused on developing more effective and advanced methods for an integral equation, and integro-differential equations such as the combined Adomian decomposition method (ADM) with modified Laplace transform for solving the nonlinear VolterraFredholm integro-differential equations (NVFIDEs) [13]. The system of Fredholm integral equations (FIEs) of the second kind with the symmetric kernel was solved [22] by using some numerical methods. Many other authors have studied nonlinear equation solutions using various methods, e.g., the solution of FIEs via the

Email addresses: ombraah20016@gmail.com (Fawziah M. Al-saar), drkp.ghadle@gmail.com (Kirtiwant P. Ghadle) 
ADM with its modification is obtained in [4], the solution of NVFIEs is found by Taylor series and hybrid of block-pulse functions in [20, 12, The system of FIEs of the second kind was solved by ADM and MADM, see [5]. ADM was applied in [7] to find the solution of linear system Volterra equations. The authors in [11] used modified ADM for solving the fuzzy NVFIEs. whereas, the authors in [19] compared the projection method with ADM to find the solution of the system of integral equations. In [23], the efficient algorithms have been used to solve Abel-type singular integral equations. Two-dimensional NVFIDEs were solved via iterative methods, see [8].

In [21], the authors employed the solution of two-dimensional NVFIEs based on the variational iteration method (VIM), the modified homotopy perturbation method (MHPM) and VIM were applied to solve the nonlinear mixed VFIE, for more details see [10, 28]. The solution of the nonlinear system of mixed VFIEs was obtained by VIM in [24]. An existence result for fractional integro-differential equations on Banach space has been studied in [25]. Singular fractional differential equations with $\psi$-Caputo operator and modified Picard's iterative method are presented in [26]. NVFIE with a generalized singular kernel and nonlinear mixed integral equations with singular Volterra kernel have been discussed in [16, 17. Monotone iterative sequences for nonlinear integro-differential equations of second order are used in [3]. Finding numerical solutions to integrodifferential equations based on Legendre multi-wavelets collocation using the new method have been studied in [18]. Some new uniqueness results of solutions to nonlinear fractional integro-differential equations have been emplemented in [1]. Legendre multi-wavelets collocation method for the numerical solution of linear and nonlinear integral equations are presented in [6]. Nonlocal problems for fractional integro-differential equation in Banach space were studied in [2].

According to the above discussions, in this paper, we apply different methods as MHPM, MADM, and VIM for solving the nonlinear mixed Volterra-Fredholm integral equation (NMVFIE) that is

$$
y(\varkappa, t)=f(\varkappa, t)+\int_{a}^{t} \int_{\Omega} F(\varkappa, t, \xi, \tau, y(\xi, \tau)) d \xi d \tau, \quad(\varkappa, t) \in \Omega \times[0, T]
$$

where $y(\varkappa, t)$ is an unknown function, $f(\varkappa, t)$ and $F(\varkappa, t, \xi, \tau, y(\xi, \tau))$ are analytic functions on $D=\Omega \times[0, T]$, $\Omega$ is a closed subset of $R^{n}, n=1,2,3$, and $R$ is the real number set.

The main motive for this paper is to develop the applications of the proposed methods in nonlinear problems with their system because these methods are the most convenient for solving such types of equations, especially the NMVFIEs. Consequently, we apply MHPM, VIM and MADM for solving the equation (1) and HPM, VIM, and ADM for solving the equation (1) and the system given in (28). Numerical examples are provided to find the exact and approximate solutions. Moreover, we use the absolute error table and comparisons with current approaches to show the accuracy and effectiveness of these methods. Finaly, we prove the convergence of the solution and the uniqueness of our proposed methods.

The rest of the article will be organized as follows: in section 2 we introduce the solution of nonlinear mixed Volterra-Fredholm integral equations using the proposed methods and their applications. The systems of the proposed methods are presented in section 3. We prove the uniqueness and existence of the solution of Eq. (1) in Section 4. In section 5, we demonstrate that the proposed methods are accurate, efficient, and readily implemented through numerical examples. Section 6 concludes this article with a brief conclusion.

\section{Description of the methods}

In this section, we briefly highlighted the key points of each proposed method for solving NMVFIE, where details can be found in [4, 11, 13, 10, 9, 28, 8].

\subsection{Homotopy perturbation method (HPM)}

Consider the general form, integral equation $L y=0$ where $L$ is an integral operator. Define a convex homotopy $H(v, p)$ as:

$$
(1-p) F(v)+p L(v)=H(v, p)=0, \quad p \in[0,1],
$$


where $F(v)$ is a functional operator with a solution $v$ that could be easily established.

Now, we know that

$$
F(v)=H(v, 0)=0, \quad H(v, 1)=L(v)=0,
$$

where the procedure of changing $p$ from 0 to 1 is just that of changing $v$ from $v_{0}$ to $y$. This is called disfigurement in topology; $F(v)$ and $L(v)$ are called homotopies.

The embedding parameter $p$ could be used as a small parameter, depending on a HPM. The solution of (2) as a power series in $p$ can be written as:

$$
v=y_{0}+p y_{1}+p^{2} y_{2}+\ldots
$$

the approximation solution of $L y=0$ when $p \rightarrow 1$ is defined by

$$
y=\lim _{p \rightarrow 1} v=y_{0}+y_{1}+y_{2}+\ldots
$$

In most cases, the series (5) is converging. On the other hand, the convergence rate is determined by the nonlinear operator $L$, see [15].

\subsection{Modified homotopy perturbation method (MHPM)}

Based on an HPM, we are constructing the form of homotopy for Eq. (1) as follows:

$$
H(v, p)=v(\varkappa, t, p) f(\varkappa, t) p \int_{0}^{t} \int_{\Omega} F(\varkappa, t, \xi, \tau, v(\xi, \tau, p)) d \xi d \tau=0 .
$$

We expand $v(\varkappa, t, p)$ by using the HPM to the form:

$$
v(\varkappa, t, p)=y_{0}(\varkappa, t)+p y_{1}(\varkappa, t)+p^{2} y_{2}+\ldots
$$

The approximation solution is

$$
y(\varkappa, t)=\lim _{p \rightarrow 1} v(\varkappa, t, p)=y_{0}(\varkappa, t)+y_{1}(\varkappa, t)+y_{2}(\varkappa, t)+\ldots
$$

Putting (7) into (6) gives

$$
\begin{aligned}
& p^{0}: y_{0}(\varkappa, t)=f(\varkappa, t) \\
& p^{i+1}: y_{i+1}(\varkappa, t)=\int_{0}^{t} \int_{\Omega} H_{i}\left(\varkappa, t, \xi, \tau, y_{0}(\xi, \tau), y_{1}(\xi, \tau), \ldots, y_{i+1}(\xi, \tau)\right) d \xi d \tau, \\
& i=0,1,2, \ldots
\end{aligned}
$$

and

$$
\begin{aligned}
H_{i}\left(\varkappa, t, \xi, \tau, y_{0}, y_{1}, \ldots, y_{i}\right) & =\left.\frac{1}{i} \frac{d^{i}}{d p^{i}} F\left(\varkappa, t, \xi, \tau, \sum_{k=0}^{\infty} p^{k} y_{k}\right)\right|_{p=0} \\
& =\left.\frac{1}{i} \frac{d^{i}}{d p^{i}} F\left(\varkappa, t, \xi, \tau, \sum_{k=0}^{i} p^{k} y_{k}\right)\right|_{p=0}
\end{aligned}
$$

The solution obtained by using (8) provides the best approximation for some strongly nonlinear problems only at a local interval. To resolve this problem, we modify the HPM as the following:

Partition the interval $[0, T]$ to $N$ subintervals $\left[t_{j}, t_{j+1}\right], j=0,1,2, \ldots, N-1$, with $t_{0}=0, t_{N}=T$. 
On the interval $\left[t_{0}, t_{1}\right]$, let

$$
\begin{aligned}
& y_{1,0}(\varkappa, t)=f(\varkappa, t), \quad t_{0} \leq t \leq t_{1}, \varkappa \in \Omega \\
& y_{1, j+1}(\varkappa, t)=\left.\frac{1}{j !} \int_{t_{0}}^{t} \int_{\Omega} \frac{d^{j}}{d p^{j}} F\left(\varkappa, t, \xi, \tau, \sum_{k=0}^{j} p^{k} y_{1, k}(\xi, \tau)\right)\right|_{p=0} d \xi d \tau,
\end{aligned}
$$

where $j=0,1,2, \ldots,(n-1)$. As a result, we get the $n$-term approximation $y_{1, n}(\varkappa, t)=\sum_{k=0}^{n}(\varkappa, t) y_{1, k}$ on $\left[t, t_{1}\right]$.

On the interval $\left[t_{1}, t_{2}\right]$, let

$$
\begin{aligned}
& y_{2,0}(\varkappa, t)=f(\varkappa, t)+\int_{t_{0}}^{t_{1}} \int_{\Omega} F\left(\varkappa, t, \xi, \tau, y_{1, n}(\xi, \tau)\right) d \xi d \tau, \quad t_{1} \leq t \leq t_{2}, \varkappa \in \Omega \\
& y_{2, j+1}(\varkappa, t)=\left.\frac{1}{j !} \int_{t_{1}}^{t} \int_{\Omega} \frac{d^{j}}{d p^{j}} F\left(\varkappa, t, \xi, \tau, \sum_{k=0}^{j} p^{k} y_{2, k}(\xi, \tau)\right)\right|_{p=0} d \xi d \tau,
\end{aligned}
$$

where $j=0,1,2, \ldots,(n-1)$. As a result, we get the $n$-term approximation $y_{2, n}(\varkappa, t)$ on $\left[t_{1}, t_{2}\right]$.

In the same way, on the interval $\left[t_{i-1}, t_{i}\right], i=3,4, \ldots, N$, let

$$
\begin{aligned}
& y_{i, 0}(\varkappa, t)=f(\varkappa, t)+\sum_{k=1}^{i-1} \int_{t_{k-1}}^{t_{k}} \int_{\Omega} F\left(\varkappa, t, \xi, \tau, y_{k, n}(\xi, \tau)\right) d \xi d \tau, \quad t_{i-1} \leq t \leq t_{i}, \varkappa \in \Omega \\
& y_{i, j+1}(\varkappa, t)=\left.\frac{1}{j !} \int_{t_{i-1}}^{t} \int_{\Omega} \frac{d^{j}}{d p^{j}} F\left(\varkappa, t, \xi, \tau, \sum_{k=0}^{j} p^{k} y_{i, k}(\xi, \tau)\right)\right|_{p=0} d \xi d \tau,
\end{aligned}
$$

where $j=0,1,2, \ldots, n-1$. As a result, we obtain the $n$-term approximation $y_{i, n}(\varkappa, t)$ on $\left[t_{i-1}, t_{i}\right]$. Therefore, the approximation solution of (11) can be obtained according to (11), (12) and $(13)$ on the interval $[0, T]$.

\subsection{Variational iteration method (VIM)}

We have another type of the NMVFIE that is given as

$$
y(\varkappa)=f(\varkappa)+\lambda_{1} \int_{0}^{\varkappa} K_{1}(\varkappa, t) F(y, t) d t+\lambda_{2} \int_{0}^{1} K_{2}(\varkappa, t) G(y, t) d t, \quad 0 \leq \varkappa, t \leq 1,
$$

where $K_{1}(\varkappa, t), K_{2}(\varkappa, t)$ are the kernels and the function $f(\varkappa)$ on the interval $0 \leq \varkappa, t \leq 1$ which are supposed to be in $L^{2}(R)$.

Now, we are solving (1) and (14) approximately by VIM. Consider the general nonlinear formula as:

$$
L(y, t)+N(y, t)=g(t)
$$

where $g(t)$ is a known analytical function, $L$ and $N$ are a linear and nonlinear operator respectively. The VIM is constructing an iterative sequence called functional correction as follows:

$$
y_{n+1}(t)=y_{n}(t)+\int_{0}^{t} \lambda\left(L\left(y_{n}, \xi\right)+N\left(\tilde{y_{n}}, \xi\right)-g(\xi)\right) d \xi
$$

where $\lambda$ is the general multiplier of Lagrange, that could be identified optimally by the variational theory, $\left(\tilde{y_{n}}, \xi\right)$ is defined as the restricted variation, i.e. $\delta \tilde{y_{n}}=0$, and $n$ indicates the $n$th iteration.

Consider nonlinear mixed integral equations are given in Eqs. (1) and (14) as a solution with $\Omega=[0,1]$.

For Eq. (1), firstly we take a partial derivative with respect to $t$ as follows 


$$
\frac{\partial y}{\partial t}-\frac{\partial f}{\partial t}-\int_{0}^{1} F(\varkappa, t, \xi, \tau, y(\xi, \tau)) d \xi-\int_{0}^{t} \int_{0}^{1} \frac{\partial F}{\partial t} d \xi d t=0
$$

Consider

$$
-\int_{0}^{1} F(\varkappa, t, \xi, \tau, y(\xi, \tau)) d \xi-\int_{0}^{t} \int_{0}^{1} \frac{\partial F}{\partial t} d \xi d t=0 .
$$

Using the VIM in the trend $t$ of a restricted variation. Then we get the following iteration sequence

$$
\begin{aligned}
& y_{n+1}(\varkappa, t)=y_{n}(\varkappa, t)+\int_{0}^{t} \lambda\left[\frac{\partial y_{n}}{\partial \tau}(\varkappa, \tau) \frac{\partial f}{\partial \tau}(\varkappa, \tau)\right. \\
& \left.-\int_{0}^{1} F(\varkappa, \tau, \xi, \tau) y(\xi, \tau) d \xi-\int_{0}^{\tau} \int_{0}^{1} \frac{\partial F}{\partial \tau} d \xi d t\right] d \tau .
\end{aligned}
$$

For the independent variable $y_{n}$, we take the variation and observe that $\delta y_{n}=0$, it follows that

$$
\delta y_{n+1}=\delta y_{n}+\left.\lambda \delta y_{n}\right|_{\tau=t}-\int_{0}^{t} \lambda^{\prime} \delta y_{n} d \tau=0 .
$$

Applying stationary conditions as the following:

$$
1+\left.\lambda(\tau)\right|_{\tau=t}=0,\left.\quad \lambda^{\prime}(\tau)\right|_{\tau=t}=0 .
$$

As a result, the Lagrange multiplier can be identified as $\lambda=-1$. Thus, we find the iteration formula as follows:

$$
\begin{aligned}
& y_{n+1}(\varkappa, t)=y_{n}(\varkappa, t)-\int_{0}^{t}\left[\frac{\partial y_{n}}{\partial \tau}(\varkappa, \tau) \frac{\partial f}{\partial \tau}(\varkappa, \tau)\right. \\
& \left.-\int_{0}^{1} F(\varkappa, \tau, \xi, \tau, y(\xi, \tau)) d \xi-\int_{0}^{\tau} \int_{0}^{1} \frac{\partial F}{\partial \tau} d \xi d t\right] d \tau .
\end{aligned}
$$

Now, for Eq. 14), put $z(\varkappa)$ is a function such that $z^{\prime}(\varkappa)=t(\varkappa)$, and noting the continuity of $t(\varkappa)$. Therefore, we get

Consider

$$
z^{\prime}(\varkappa)=f(\varkappa)+\lambda_{1} \int_{0}^{\varkappa} k_{1}(\varkappa, t) F\left(z^{\prime}, t\right) d t+\lambda_{2} \int_{0}^{1} k_{2}(\varkappa, t) G\left(z^{\prime}, t\right) d t .
$$

$$
\lambda_{1} \int_{0}^{\varkappa} k_{1}(\varkappa, t) F\left(z^{\prime}, t\right) d t+\lambda_{2} \int_{0}^{1} k_{2}(\varkappa, t) G\left(z^{\prime}, t\right) d t,
$$

we have an iteration sequence as a bound variation

$$
\begin{aligned}
z(n+1)= & z(n)+\int_{0}^{\varkappa} \lambda\left[z_{n}^{\prime}(\xi)-\lambda_{1} \int_{0}^{\xi} k_{1}(\xi, t) F\left(z^{\prime}(n), t\right) d t\right. \\
& \left.-\lambda_{2} \int_{0}^{\xi} k_{2}(\xi, t) G\left(z^{\prime}(n), t\right) d t-f(\xi)\right] d \xi .
\end{aligned}
$$

For the independent variable $z_{n}$, we take the variation and observing that $\delta z_{n}(0)=0$, it follows that

$$
\delta z_{n+1}=\delta z_{n}+\left.\lambda(\xi) \delta z_{n}\right|_{\xi=\varkappa}-\int_{0}^{\varkappa} \lambda^{\prime}(\xi) \delta z_{n} d \xi=0 .
$$


By applying stationary conditions, we have

$$
1+\left.\lambda(\xi)\right|_{\xi=\varkappa}=0,\left.\quad \quad \lambda^{\prime}(\xi)\right|_{\xi=\varkappa}=0 .
$$

Thus, the general multiplier of Lagrange easily can be identified as $\lambda=-1$. So, we find the iteration formula as follows:

$$
\begin{aligned}
z(n+1)= & z(n)-\int_{0}^{\varkappa}\left[z_{n}^{\prime}(\xi)-\lambda_{1} \int_{0}^{\xi} k_{1}(\xi, t) F\left(z^{\prime}(n), t\right) d t\right. \\
& \left.-\lambda_{2} \int_{0}^{\xi} k_{2}(\xi, t) G\left(z^{\prime}(n), t\right) d t-f(\xi)\right] d \xi .
\end{aligned}
$$

\subsection{Adomian decomposition method (ADM)}

Consider the differential equation:

$$
L y+R y+N y=g(\varkappa, t),
$$

where $g(\varkappa, t)$ represents the source term, $L$ and $N$ indicate the highest order linear derivative and the nonlinear terms respectively, and the linear differential operator of order smaller than $L$ is denoted by $R$. If we apply the linear inverse operator $L$ to both sides of Eq. (18) then we get

$$
y=f(\varkappa, t)-L^{-1}(R y(\varkappa, t))+L^{-1}(N y(\varkappa, t)),
$$

where $f(\varkappa, t)$ denotes the terms obtained by integrating $g(\varkappa, t)$ and applying the given conditions, which have all been presumed to be specified. The ADM of the integral equation (1) introduces the series as the following:

$$
y(\varkappa, t)=\sum_{i=0}^{\infty} y_{i}(\varkappa, t),
$$

where $y(\varkappa, t)$ represents the solution of Eq. (1), and $y_{i}(\varkappa, t)$ are the components that have been determined recurrently.

The components $y_{0}, y_{1}, y_{2}, \ldots$, are recursively determined through using the formula

$$
\begin{aligned}
& y_{0}(\varkappa, t)=f(\varkappa, t) \\
& y_{i+1}(\varkappa, t)=-L^{-1}\left(R y_{i}\right)-L^{-1}\left(N y_{i}\right), \quad i \geq 0,
\end{aligned}
$$

which leads us to determine the $y$ components. After the determination of the components $y_{0}, y_{1}, y_{2}, \ldots y_{n}$, the solution $y$ in the form of a series defined by Eq. (22) immediately follows:

$$
y=y_{0}+y_{1}+y_{2}+\ldots
$$

Observe that the ADM indicates that the function $f(\varkappa, t)$ as considered above denies the zeroth component $y_{0}$.

\subsection{Modified Adomian decomposition method (MADM)}

Recently, Wazwaz in [27] developed a reliable solution of the ADM, and its efficiency has been explicitly supported in numerous studies. For applying this modification, we divide the function $f$ into the sum of two parts $f_{0}$ and $f_{1}$ as follows:

$$
f=f_{0}+f_{1}
$$


Suggesting only a small variation on the components $y_{0}$ and $y_{1}$, the variation that we suggest is that only the part $f_{0}$ be assigned to the zeroth component $y_{0}$, while the terms are given in 21) will be composited with the part $f_{1}$ to determine $y_{1}$. Under these suggestions, the recursive modificaion is formulated as the following:

$$
\begin{aligned}
& y_{0}(\varkappa, t)=f(\varkappa, t) \\
& y_{1}(\varkappa, t)=f_{1}(\varkappa, t)-L^{-1}\left(R y_{0}\right)-L^{-1}\left(N y_{0}\right), \\
& y_{i+2}(\varkappa, t)=L^{-1}\left(R y_{i+1}\right)-L^{-1}\left(N y_{i+1}\right), \quad i \geq 0 .
\end{aligned}
$$

This method determines the nonlinear function $F(\varkappa, t, \xi, \tau, y(\xi, \tau))$ through an infinite sequence of polynomials

$$
F(\varkappa, t, \xi, \tau, y(\xi, \tau))=\sum_{n=0}^{\infty} A_{n},
$$

where $A_{n}$ are called the Adomian polynomials that symbolize to the nonlinear term $F(\varkappa, t, \xi, \tau, y(\xi, \tau))$ and which could be calculated for different nonlinear operators classes.

Now, by substituting $(24)$ and $(23)$ into 22$)$ we get

$$
\sum_{n=0}^{\infty} y_{i}(\varkappa, t)=f(\varkappa, t)+\int_{0}^{t} \int_{\Omega}\left(\sum_{n=0}^{\infty} A_{n}\right) d \xi d \tau .
$$

Assume that $f(\varkappa, t)$ is decomposed into the sum of $f_{0}$ and $f_{1}$ as follows:

$$
f(\varkappa, t)=f_{0}(\varkappa, t)+f_{1}(\varkappa, t) .
$$

The components $y_{i}(\varkappa, t), n \leq 0$ will be identified in a recursive way. This can be completed through assigning $f_{0}(\varkappa, t)$ to the component $y_{0}(\varkappa, t)$ while the terms are given in $(26)$ will be composeted with the part $f_{1}(\varkappa, t)$ to the component $y_{1}(\varkappa, t)$. Therefore, the MADM provides the recursive formula

$$
\begin{aligned}
& y_{0}(\varkappa, t)=f(\varkappa, t), \\
& y_{1}(\varkappa, t)=f_{1}(\varkappa, t)+\int_{0}^{t} \int_{\Omega} A_{0} d \xi d \tau, \\
& y_{i+2}(\varkappa, t)=\int_{0}^{t} \int_{\Omega} A_{i+1} d \xi d \tau, \quad i \geq 0 .
\end{aligned}
$$

Relation (27) will allow us to define the components $y_{n}(\varkappa, t), n \geq 0$ recurrently. As consequence of that, the $y(\varkappa, t)$ sequence solution is easily available. Already, it has been stated that the MADM could be combined with the noise terms phenomenon to find the fast convergence of the solution. Specifically, this phenomenon can extend a solution that prevents complicated computing of Adomian polynomials. Generally, integrating the noise term phenomenon with the modified decomposition approach offers an encouraging technique for treating differential and integral equations.

\section{Description of the methods for solving NMSVFIEs}

We will offer a brief highlight as the prime point of every method in this section to find the solution for the nonlinear mixed Volterra-Fredholm integral equations (NMSVFIEs), to find out more details see [5, 7, 24, 27].

We introduce the nonlinear mixed system of Volterra-Fredholm as Eq (1), where

$$
\begin{aligned}
y(\varkappa, t)=\left(y_{1}(\varkappa, t)+\right. & \left.y_{2}(\varkappa, t)+\cdots+y_{n}(\varkappa, t)\right)^{t} \\
f(\varkappa, t)=\left(f_{1}(\varkappa, t)+\right. & \left.f_{2}(\varkappa, t)+\cdots+f_{n}(\varkappa, t)\right)^{t} \\
F(\varkappa, t, \xi, \tau) y(\xi, \tau)= & \left(F_{1}(\varkappa, t, \xi, \tau, y(\xi, \tau))+F_{2}(\varkappa, t, \xi, \tau, y(\xi, \tau))\right. \\
& \left.+\cdots+F_{n}(\varkappa, t, \xi, \tau, y(\xi, \tau))\right)^{t},
\end{aligned}
$$


where the unknown functions $y(\varkappa, t)$ is defined on $D=[0, T] \times \Omega$, and a closed subset $\Omega$ is defined on $\left(R^{n}\right)$, $n=1,2,3$.

\section{1. $H P M$}

To demonstrate the HPM for NMSVFIEs, we consider the system (1) and (28) as follows

$$
y_{i}(\varkappa, t)=f_{i}(\varkappa, t)+\int_{0}^{t} \int_{\Omega} k_{i}\left(\varkappa, t, \xi, \tau, y_{1}(\xi, \tau), \ldots, y_{n}(\xi, \tau)\right) d \xi d \tau, \quad i=1,2, \ldots, n .
$$

Now, we divide the function $f_{i}$ into $f_{i, 0}$ and $f_{i, 1}$ and the sum of these two parts can be written as:

$$
f_{i}=f_{i, 0}+f_{i, 1}, \quad i=1,2, \ldots, n
$$

Rewriting Eq. 29) as

$$
y_{i}(\varkappa, t)=f_{i, 0}(\varkappa, t)+f_{i, 1}(\varkappa, t)+\int_{0}^{t} \int_{\Omega} k_{i}\left(\varkappa, t, \xi, \tau, y_{1}(\xi, \tau), \ldots, y_{n}(\xi, \tau)\right) d \xi d \tau, \quad i=1,2, \ldots, n .
$$

To solve the Eq. 29, we will use the HPM to presented two homotopies cases as follows

\section{Case 1.}

$$
\begin{aligned}
& F_{i}(\varkappa, t)-f_{i}(\varkappa, t)-p\left(F_{i}(\varkappa, t)-f_{i}(\varkappa, t)\right. \\
& \left.-\int_{\Omega} K_{i}\left(\varkappa, t, \xi, \tau, F_{1}(\xi, \tau), \ldots, F_{n}(\xi, \tau)\right) d \xi d \tau\right)=0, \quad i=1,2, \ldots, n .
\end{aligned}
$$

Assume that the solution of the system 32 is defined by

$$
F_{i}(\varkappa, t)=F_{i, 0}(\varkappa, t)+p F_{i, 1}(\varkappa, t)+p^{2} F_{i, 2}(\varkappa, t)+\ldots, \quad i=1,2, \ldots, n,
$$

where the functions $F_{i, j}(\varkappa, t), i=1,2, \ldots, n, \quad j=0,1,2, \ldots$, must be determined.

Putting Eq. (32) into Eq. (32), and according to on powers of $p$, we reordered the terms, we get:

$$
\begin{aligned}
& p^{0}: F_{i, 0}(\varkappa)=f_{1}(\varkappa), \quad i=1,2, \ldots, n, \\
& p^{1}: F_{i, 1}(\varkappa)=\int_{a}^{t} \int_{\Omega} k_{i}\left(\varkappa, t, \xi, \tau, F_{1,0}(\xi, \tau), F_{2,0}(\xi, \tau), \ldots, F_{n, 0}(\xi, \tau)\right) d \xi \tau, \\
& p^{2}: F_{i, 2}(\varkappa)=\int_{a}^{t} \int_{\Omega} k_{i}\left(\varkappa, t, \xi, \tau, F_{1,1}(\xi, \tau), F_{2,1}(\xi, \tau), \ldots, F_{n, 1}(\xi, \tau)\right) d \xi \tau, \\
& \vdots \\
& p^{k}: F_{i, k}(\varkappa)=\int_{a}^{t} \int_{\Omega} k_{i}\left(\varkappa, t, \xi, \tau, F_{1, k}(\xi, \tau), F_{2, k}(\xi, \tau), \ldots, F_{n, k}(\xi, \tau)\right) d \xi \tau, \\
& \vdots \\
& i=2, \ldots, n .
\end{aligned}
$$

Therefore by setting $p=1$ in Eq. (30) we can obtain the following approximation solutions

$$
y_{i}(\varkappa, t)=\lim _{p \rightarrow 1} F_{i}(\varkappa, t)=\sum_{j=0}^{\infty} F_{i, j}(\varkappa, t), \quad i=1,2, \ldots, n .
$$




\section{Case 2.}

$$
\begin{aligned}
& y_{i}(\varkappa, t)-f_{i, 0}(\varkappa, t)-p\left(f_{i, 1}(\varkappa, t)\right. \\
& \left.+\int_{0}^{t} \int_{\Omega} K_{i}\left(\varkappa, t, \xi, \tau, y_{1}(\xi, \tau), \ldots, y_{n}(\xi, \tau)\right) d \xi d \tau\right)=0, \quad i=1,2, \ldots, n .
\end{aligned}
$$

Assume that the solution of the system $(35)$ is given by

$$
y_{i}(\varkappa, t)=y_{i, 0}(\varkappa, t)+p y_{i, 1}(\varkappa, t)+p^{2} y_{i, 2}(\varkappa, t)+\ldots, \quad i=1,2, \ldots, n,
$$

where the functions $y_{i, j}(\varkappa, t), \quad i=1,2, \ldots, n, \quad j=1,2, \ldots, n$, must be defined.

Putting Eq. (35) into Eq. (35), and according to on powers of $p$, we reordered the terms, we obtain

$$
\begin{aligned}
& p^{0}: y_{i, 0}(\varkappa)=f_{i, 0}(\varkappa, t), \quad i=1,2, \ldots, n, \\
& p^{1}: y_{i, 1}(\varkappa)=f_{i, 0}(\varkappa, t)+\int_{a}^{t} \int_{\Omega} k_{i}\left(\varkappa, t, \xi, \tau, y_{1,0}(\xi, \tau), y_{2,0}(\xi, \tau), \ldots, y_{n, 0}(\xi, \tau)\right) d \xi \tau, \\
& p^{2}: y_{i, 2}(\varkappa)=\int_{a}^{t} \int_{\Omega} k_{i}\left(\varkappa, t, \xi, \tau, y_{1,1}(\xi, \tau), y_{2,1}(\xi, \tau), \ldots, y_{n, 1}(\xi, \tau)\right) d \xi \tau, \\
& \vdots \\
& p^{k}: y_{i, k}(\varkappa)=\int_{a}^{t} \int_{\Omega} k_{i}\left(\varkappa, t, \xi, \tau, y_{1, k}(\xi, \tau), y_{2, k}(\xi, \tau), \ldots, y_{n, k}(\xi, \tau)\right) d \xi \tau, \\
& \vdots \\
& i=2, \ldots, n .
\end{aligned}
$$

Therefore, by setting $p=1$ in Eq. 29, we can obtain the following approximation solution

$$
y_{i}(\varkappa, t)=\lim _{p \rightarrow 1} y_{i}(\varkappa, t)=\sum_{j=0}^{\infty} y_{i, j}(\varkappa, t), \quad i=1,2, \ldots, n .
$$

\section{2. $V I M$}

To find the solution of Eqs. (1) and (28) by VIM, we first consider $i$ th of Eq.(1) as follows

$$
y_{i}(\varkappa, t)=f_{i}(\varkappa, t)+\int_{0}^{t} \int_{\Omega} f_{i}\left(\varkappa, t, \xi, \tau, y_{1}(\xi, \tau), \ldots, y_{1}(\xi, \tau)\right) d \xi d \tau, \quad i=1,2, \ldots, n .
$$

Now, we are trying to get an effective method to solve a nonlinear system in Eq. (28). Assume $\Omega=[0,1]$, then we take the partial derivative of Eq. (37) concerning $t$ as the following

$$
\frac{\partial y_{i}(\varkappa, t)}{\partial t}-\frac{\partial f_{i}(\varkappa, t)}{\partial t}-\int_{0}^{1} F_{i}\left(\varkappa, t, \xi, \tau, y_{1}(\xi, \tau), \ldots, y_{n}(\xi, \tau)\right) d \xi-\int_{0}^{t} \int_{0}^{1} \frac{\partial F_{i}}{\partial t} d \xi d t=0 .
$$

As a restricted variation that we apply VIM for Eq. 38, thus we get the iteration sequences of the system as

$$
\begin{aligned}
& y_{i, n+1}(\varkappa, t)=y_{i, n}(\varkappa, t)+\int_{0}^{t} \lambda_{i}\left[\frac{\partial f_{i}}{\partial \tau}(\varkappa, \tau) \frac{\partial y_{i, n}}{\partial \tau}(\varkappa, \tau)\right. \\
& \left.-\int_{0}^{1} F_{i}\left(\varkappa, \tau, \xi, \tau, y_{1}(\xi, \tau), \ldots, y_{n}(\xi, \tau)\right) d \xi-\int_{0}^{\tau} \int_{0}^{1} \frac{\partial F_{i}}{\partial \tau} d \xi d t\right] d \tau, \\
& i=1,2, \ldots, n \quad n=1,2, \ldots
\end{aligned}
$$


Taking the effect $\delta$ and the variation theorem in both sides of Eq. 39 and also assuming

$$
\begin{aligned}
& \delta y_{i, n+1}=0 \\
& \left.\delta\left(-\int_{0}^{1} F_{i}\left(\varkappa, \tau, \xi, \tau, y_{1}(\xi, \tau), \ldots, y_{n}(\xi, \tau)\right) d \xi-\int_{0}^{\tau} \int_{0}^{1} \frac{\partial F_{i}}{\partial \tau} d \xi d t\right] d \tau\right)=0
\end{aligned}
$$

we get

$$
\delta y_{i, n+1}(\varkappa, t)=\left(1+\lambda_{i}(t)\right) \delta y_{i, n}(\varkappa, t)-\lambda_{i}^{\prime}(\tau) \delta y_{i, n}(\xi, \tau) d \tau=0 .
$$

Through considering $\delta y_{i, n+1}(\varkappa, t)=0$, by applying the stationary conditions, we have

$$
1+\left.\lambda_{i}(t)\right|_{\tau=t}=0,\left.\quad \quad \lambda_{i}^{\prime}(\tau)\right|_{\tau=t}=0 .
$$

Therefore, the general multiplier of Lagrange could be identified easily as $\lambda_{i}=-1$.

By substituting $\lambda_{i}=-1$, in Eq. (39), then to finding the solution of Eqs. (1) and (28), we get the following iteration algebraic system

$$
\begin{aligned}
& y_{i, n+1}(\varkappa, t)=y_{i, n}(\varkappa, t)-\int_{0}^{t}\left[\frac{\partial y_{i, n}(\varkappa, t)}{\partial \tau}(\varkappa, \tau) \frac{\partial f_{i}(\varkappa, t)}{\partial \tau}(\varkappa, \tau)\right. \\
& \left.-\int_{0}^{1} F_{i}\left(\varkappa, \tau, \xi, \tau, y_{1}(\xi, \tau), \ldots, y_{n}(\xi, \tau)\right) d \xi-\int_{0}^{\tau} \int_{0}^{1} \frac{\partial F_{i}}{\partial \tau} d \xi d t\right] d \tau .
\end{aligned}
$$

\section{3. $A D M$}

In this part, we will show how to use the ADM to solve the NMSVFIEs:

In ADM the canonical form of (1) for Eqs. (1) and (29), can be given by

$$
y_{i}(\varkappa, t)=f_{i}(\varkappa, t)+N_{i}(\varkappa, t) .
$$

Then it can be decomposed into nonlinear and linear and components, depending on the integral operator $N_{i}$ 's features, where $N_{i}$ is an analytical operator of the nonlinear integral, so we have

$$
N_{i}(\varkappa, t)=\int_{0}^{t} \int_{\Omega} k_{i}\left(\varkappa, t, \xi, \tau, y_{1}(\xi, \tau), \ldots, y_{n}(\xi, \tau)\right) d \xi d \tau, \quad i=1,2, \ldots, n .
$$

To implement the ADM, let $y_{i}(\varkappa, t)=\sum_{j=0}^{\infty} N_{i, j}(\varkappa, t)$ and $N_{i}(\varkappa, t)=\sum_{j=0}^{\infty} A_{i, j}(\varkappa, t)$ where $A_{i, j}, j=$ $0,1,2, \ldots$ are polynomials based on

$y_{10}, \ldots, y_{1 j}, \ldots, y_{n 0}, \ldots, y_{n j}$ that called Adomian polynomials and then we approximate the solution by $\varphi_{i k}(\varkappa, t)=\sum_{j=0}^{k-1} y_{i j}(\varkappa, t)$ where $\lim _{k \rightarrow \infty} \varphi_{i k}(\varkappa, t)=y_{i}(\varkappa, t)$, and we get

$$
y_{i}(\varkappa, t)=\sum_{j=0}^{\infty} y_{i j}(\varkappa, t) \lambda^{j} .
$$

To find the Adomian polynomials we can write:

$$
\begin{aligned}
y_{i}(\varkappa, t) & =\sum_{j=0}^{\infty} y_{i j}(\varkappa, t) \lambda^{j}, \\
N_{i}(\varkappa, t) & =\sum_{j=0}^{\infty} A_{i j}(\varkappa, t) \lambda^{j},
\end{aligned}
$$

where $\lambda$ is a parameter established for convenience. We have got from 44

$$
A_{i j}(\varkappa, t)=\frac{1}{j !}\left[\frac{d^{j}}{d \lambda^{j}} N_{i \lambda}\left(y_{1}, \ldots, y_{n}\right)\right]_{\lambda=0} .
$$


As a result, we use the following Adomian scheme

$$
\begin{gathered}
y_{i, 0}(\varkappa, t)=f_{i}(\varkappa, t), \\
y_{i, j+1}(\varkappa, t)=\frac{1}{j !} \int_{0}^{t} \int_{\Omega}\left[\frac{d^{j}}{d \lambda^{j}} g_{i}\left(\varkappa, t, \xi, \tau, \sum_{k=0}^{\infty} y_{1 k} \lambda^{k}, \sum_{k=0}^{\infty} y_{2 k} \lambda^{k}\right)\right]_{\lambda=0} d \xi d \tau .
\end{gathered}
$$

\section{Theoretical Results}

In this section, we prove the uniqueness and existence theorems for the NMVFIEs.

\section{Lemma 4.1.}

$$
\begin{aligned}
\left.\frac{1}{n !} \frac{d^{n}}{d p^{n}} F\left(\varkappa, t, \xi, \tau, \sum_{k=0}^{n} p^{k} y_{i, k}\right)\right|_{p=0} & =y_{i, n} \partial_{v} F\left(\varkappa, t, \xi, \tau, y_{i, 0}\right) \\
& +\frac{1}{2} \sum_{i_{1}+i_{2}=n, i_{1}, i_{2} \geq 1} y_{i, i_{1}} y_{i, i_{2}} \partial_{v}^{2} F\left(\varkappa, t, \xi, \tau, y_{i, 0}\right) \\
& +\cdots+\frac{1}{k !} \sum_{\sum_{j=1}^{k} i_{j}=n, i_{1}, i_{j} \geq 1} y_{i, i_{1}} y_{i, i_{2}} \ldots y_{i, i_{k}} \partial_{v}^{k} F\left(\varkappa, t, \xi, \tau, y_{i, 0}\right) \\
& +\cdots+\frac{1}{n !} y_{i, i_{1}}^{n} \partial_{v}^{n} F\left(\varkappa, t, \xi, \tau, y_{i, 0}\right)
\end{aligned}
$$

where $\partial_{v} F\left(\varkappa, t, \xi, \tau, y_{i, 0}\right)=\left.\frac{\partial}{\partial_{v}} F(\varkappa, t, \xi, \tau, v)\right|_{v=y_{i, 0}}$. and

$\partial_{v}^{k} F\left(\varkappa, t, \xi, \tau, y_{i, 0}\right)=\left.\frac{\partial^{k}}{\partial_{v^{k}}} F(\varkappa, t, \xi, \tau, v)\right|_{v=y_{i, 0}}$.

Put

$$
\begin{gathered}
M_{i}=\sup \left[\max _{0 \leq r \leq t \leq 1, \varkappa, \xi \in \Omega}\left|\partial_{v^{k}} F\left(\varkappa, t, \xi, \tau, y_{i, 0}(\xi, \tau)\right)\right|, k=0,1,2, \ldots\right] \\
c_{0}=4 \frac{5}{9}, \quad c_{1}=9, \quad \beta=\frac{c_{1}}{c_{0}^{2}}\left(e^{c_{0}}-1\right), \quad \beta_{n}=\frac{c_{1}}{c_{0}^{2}} \sum_{m=1}^{n} \frac{c_{0}^{m}}{m !}, \quad q_{i}=t_{i}-t_{i-1} \\
S_{1}(k)=k^{2} \sum_{i_{1}+i_{2}=k, i_{1}, i_{2} \geq 1} \frac{1}{i_{1}^{2} i_{2}^{2}}, \quad S_{2}(k)=(k+1)^{2} \sum_{i_{1}+i_{2}=k, i_{1}, i_{2} \geq 1} \frac{1}{i_{1}^{2} i_{2}^{2}} .
\end{gathered}
$$

Lemma 4.2. $S_{1}(k) \leq c_{0}, S_{2}(k) \leq c_{1}$ for every integer $k \geq 2$.

Proof. It is not difficult to prove $S_{1}(k+1)<S_{1}(k)$ for every $k \geq 4$. So $S_{1}(k) \leq \max \left[S_{1}(2), S_{1}(3), S_{1}(4)\right]=$ $4 \frac{5}{9}=c_{0}$. For every $k \geq 4$,

$$
\begin{aligned}
S_{2}(k+1) & -S_{2}(k)=\left(\frac{k+2}{k+1}\right)^{2} S_{1}(k+1)-\left(\frac{k+1}{k}\right)^{2} S_{1}(k) \\
= & \left(1+\frac{1}{k+1}\right)^{2} S_{1}(k+1)-\left(1+\frac{1}{k}\right)^{2} S_{1}(k)<0 .
\end{aligned}
$$

Hence $S_{2}(k) \leq \max \left[S_{2}(2), S_{2}(3), S_{3}(4)\right]=9=c_{1}$.

Lemma 4.3. For every integer $k \geq l$,

$$
(k+1)^{2} . \sum_{\sum_{j=1}^{n+l} i_{j}=k, i_{j} \geq 1} \frac{1}{i_{1}^{2} i_{2}^{2} \ldots i_{l}^{2}} \leq c_{0}^{l-2} c_{1}
$$


Proof. By using the mathematical induction. When $l=2$, and combining it with lemma 4.2 , we obtain

$$
(k+1)^{2} . \sum_{\sum_{j=1}^{n+l} i_{j}=k, i_{j} \geq 1} \frac{1}{i_{1}^{2} i_{2}^{2}}=S_{2}(k) \leq c_{1} .
$$

Assume that the conclusion holds when $l=n$, that is,

$$
(k+1)^{2} . \sum_{\sum_{j=1}^{l} i_{j}=k, i_{j} \geq 1} \frac{1}{i_{1}^{2} i_{2}^{2} \ldots i_{n}^{2}}=S_{2}(k) \leq c_{0}^{n-2} c_{1} .
$$

Now for $l=n+1$,

$$
\begin{aligned}
& (k+1)^{2} \cdot \sum_{\sum_{j=1}^{n+l} i_{j}=k, i_{j} \geq 1} \frac{1}{i_{1}^{2} i_{2}^{2} \ldots i_{n+1}^{2}} \\
& =\sum_{i_{n+1}=1}^{k-n} \frac{(k+1)^{2}}{i_{n+1}^{2}\left(k-i_{n+1}+1\right)^{2}}\left(k-i_{n+1}+1\right)^{2} \cdot \sum_{\sum_{j=1}^{n} i_{j}=k-i_{n+1}, i_{j} \geq 1} \frac{1}{i_{1}^{2} i_{2}^{2} \ldots i_{n}^{2}} \\
& \leq c_{0}^{n-2} c_{1} \sum_{i_{n+1}=1}^{k} \frac{(k+1)^{2}}{i_{n+1}^{2}\left(k-i_{n+1}+1\right)^{2}}\left(k-i_{n+1}+1\right)^{2} \\
& =c_{0}^{n-2} c_{1} S_{1}(k+1) \leq c_{0}^{n-2} c_{1} c_{0}=c_{0}^{n-1} c_{1} .
\end{aligned}
$$

Put $L=\int_{\Omega} d \xi$. Without a doubt, the value of $L$ is finite.

Lemma 4.4. $\left\|y_{i, n}\right\|_{c} \leq \frac{\left(\beta q_{i} M_{i} L\right)^{n}}{n^{2}}, \quad n=1,2, \ldots$

Proof. $y_{i, 1}=\int_{t_{i-1}}^{t} \int_{\Omega} F\left(\varkappa, t, \xi, \tau, y_{i, 0}\right) d \xi d \tau$ when $n=1$. Combining this with $\int_{t_{i-1}}^{t} d \tau=t-t_{i-1} \leq t_{i}-t_{i-1}=$ $q_{i}$,

it follows that

$$
\begin{aligned}
\left|y_{i, 1}(\varkappa, t)\right| \leq & \max _{0 \leq \tau \leq t \leq 1, \varkappa, \xi \in \Omega}\left|F\left(\varkappa, t, \xi, \tau, y_{i, 0}(\xi, \tau)\right)\right| \int_{t_{i-1}}^{t} \int_{\Omega} d \xi d \tau \\
& \leq L M_{i} q_{i} \leq \beta L M_{i} q_{i} .
\end{aligned}
$$

So, $\left\|y_{i, 1}\right\|_{c} \leq \beta L M_{i} q_{i}$. Hence, the conclusion holds for $n=1$.

Now, assuming that $\left\|y_{i, n}\right\|_{c} \leq \frac{\left(\beta q_{i} M_{i} L\right)^{n}}{n^{2}}, \quad n=1,2, \ldots$ is correct for every $n \leq k$, then when $n=k+1$,

$$
\begin{aligned}
& y_{i, k+1}(\varkappa, t)=\left.\int_{t_{i-1}}^{t} \int_{\Omega} \frac{1}{k !} \frac{d^{k}}{d p^{k}} F\left(\varkappa, t, \xi, \tau, \sum_{i=0}^{k} p^{l} y_{i, 1}(\xi, \tau)\right)\right|_{p=0} d \xi d \tau \\
= & \int_{t_{i-1}}^{t} \int_{\Omega} \sum_{m=1}^{k} \frac{1}{m !} \sum_{\sum_{j=1}^{m} i_{j}=k, i_{j} \geq 1} y_{i, i_{1}}, y_{i, i_{2}}, \ldots, y_{i, i_{m}} \partial_{v}^{m} F\left(\varkappa, t, \xi, \tau, y_{i, 0}(\xi, \tau)\right) d \xi d \tau .
\end{aligned}
$$


Hence,

$$
\begin{aligned}
\left|y_{i, k+1}(\varkappa, t)\right| \leq & M_{i} \cdot \int_{\Omega} d \xi \int_{t_{i-1}}^{t} d \tau \sum_{m=1}^{k} \frac{1}{m !} \sum_{m=1}^{k} \frac{1}{m !} \sum_{\sum_{j=1}^{m} i_{j}=k, i_{j} \geq 1}\left\|y_{i, i_{1}}\right\|_{c}\left\|y_{i, i_{2}}\right\|_{c} \ldots,\left\|y_{i, i_{m}}\right\|_{c} \\
& \leq q_{i} M_{i} L \cdot \sum_{m=1}^{k} \frac{1}{m !} \sum_{m=1}^{k} \frac{1}{m !} \sum_{\sum_{j=1}^{m} i_{j}=k, i_{j} \geq 1} \frac{\left(\beta q_{i} M_{i} L\right)^{i 1}}{i_{1}^{2}} \frac{\left(\beta q_{i} M_{i} L\right)^{i 2}}{i_{2}^{2}} \ldots \frac{\left(\beta q_{i} M_{i} L\right)^{i m}}{i_{m}^{2}} \\
& \leq q_{i} M_{i} L \mid\left(\beta q_{i} M_{i} L\right) \sum_{m=1}^{k} \frac{1}{m !} \sum_{m=1}^{k} \frac{1}{m !} \sum_{\sum_{j=1}^{m} i_{j}=k, i_{j} \geq 1} \frac{1}{i_{1}^{2} i_{2}^{2} \ldots i_{m}^{2}} .
\end{aligned}
$$

Lemma (4.3) shows that

$$
\begin{aligned}
& (k+1)^{2} \sum_{m=1}^{k} \frac{1}{m !} \sum_{\sum_{j=1}^{m} i_{j}=k, i_{j} \geq 1} \frac{1}{i_{1}^{2} i_{2}^{2} \ldots i_{m}^{2}} \\
= & \sum_{m=1}^{k} \frac{1}{m !} \frac{(k+1)^{2}}{\sum_{\sum_{j=1}^{m} i_{j}=k, i_{j} \geq 1}} \frac{1}{i_{1}^{2} i_{2}^{2} \ldots i_{m}^{2}} \\
\leq & \sum_{m=1}^{k} \frac{1}{m !} c_{0}^{m-2} c_{1}=\frac{c_{1}}{c_{0}^{2}} \sum_{m=1}^{k} \frac{c_{0}^{m}}{m !} \leq \frac{c_{1}}{c_{0}^{2}}\left(e^{c_{0}}-1\right)=\beta .
\end{aligned}
$$

Consequently,

$$
\sum_{m=1}^{k} \frac{1}{m !} \sum_{\sum_{j=1}^{m}} \frac{1}{i_{j}=k, i_{j} \geq 1} \frac{\beta}{i_{1}^{2} i_{2}^{2} \ldots i_{m}^{2}} \leq \frac{\beta}{(k+1)^{2}}
$$

Hence,

$$
\left|y_{i, k+1}(\varkappa, t)\right| \leq q_{i} M_{i} L\left(\beta q_{i} M_{i} L\right)^{k} \frac{\beta}{(k+1)^{2}}=\frac{\left(\beta q_{i} M_{i} L\right)^{k+1}}{(k+1)^{2}} .
$$

Thus, $\left\|y_{i, k+1}\right\|_{c} \leq \frac{\left(\beta q_{i} M_{i} L\right)^{k+1}}{(k+1)^{2}}$. The proof is completed.

Applying Lemma (4.4), the following theorem easily can be proved.

Theorem 4.5. If $\beta q_{i} M_{i} L \leq 1, F(\varkappa, t, \xi, \tau, v)$ is not an nth-order polynomial with respect to $v$, then:

- $\sum_{k=0}^{\infty} y_{i, k}$ converges to the exact solution $Y_{i}(\varkappa, t)$ of Eq. 13.).

- The error estimation $\left\|\sum_{k=n+1}^{\infty} y_{i, k}\right\|_{c} \leq \frac{\left(\beta q_{i} M_{i} L\right)^{n+1}}{1-\beta q_{i} M_{i} L}$ with $\beta q_{i} M_{i} L<1$ and the error estimation $\left\|\sum_{k=n+1}^{\infty} y_{i, k}\right\|_{c} \leq \frac{1}{n}$ with $\beta q_{i} M_{i} L=1$.

Similarly, we can prove the following theorem:

Theorem 4.6. If $F(\varkappa, t, \xi, \tau, v)$ is not an nth-order polynomial with respect to $\beta_{n} q_{i} M_{i} L \leq 1$, then:

- $\sum_{k=0}^{\infty} y_{i, k}$ converges to the exact solution $Y_{i}(\varkappa, t)$ of Eq. 13.

- The error estimation $\left\|\sum_{k=n+1}^{\infty} y_{i, k}\right\|_{c} \leq \frac{\left(\beta q_{i} M_{i} L\right)^{n+1}}{1-\beta q_{i} M_{i} L}$ with $\beta_{n} q_{i} M_{i} L<1$ and the error estimation $\left\|\sum_{k=n+1}^{\infty} y_{i, k}\right\|_{c} \leq \frac{1}{n}$ with $\beta_{n} q_{i} M_{i} L=1$. 
Remark: If the length of interval $\left[t_{i-1}, t_{i}\right]$ is small enough, then $q_{i}$ is small enough which ensures that $\beta q_{i} M_{i} L<1$ or $\beta_{n} q_{i} M_{i} L<1$. Thus, Theorems 4.5 and 4.6 show that algorithm (13) converges to the exact solution of Eq. (1).

Theorem 4.7. Suppose that

- $G(\varkappa, t, \xi, \tau, y)=\int_{\Omega} F(\varkappa, t, s, y(s, t)) d s$.

- There exist nonnegative continuous $m(\varkappa, t)$ and $n(\tau)$ defined on $D$ and $R$ respectively, such that $G(\varkappa, t, \xi, \tau, y)$ satisfies to a generalized Lipschitz condition of the form

$$
\left\|G\left(\varkappa, t, \xi, \tau, y_{1}\right)-G\left(\varkappa, t, \xi, \tau, y_{2}\right)\right\| \leq m(\varkappa, t) n(\tau)\left\|y_{1}-y_{2}\right\| .
$$

Then, the bound for the Adomian decomposition series for Eq. (1), can be established as

$$
\left\|\sum_{k=0}^{\infty} A_{n}\right\| \leq v(\varkappa, t) m(\varkappa, t) \int_{0}^{t} v(\varkappa, t) n(\tau) \operatorname{e\varkappa p}\left[\int_{\tau}^{t} m(\varkappa, \eta) n(\eta) d \eta\right] d \tau,
$$

where

$$
v(\varkappa, t)=\left\|\int_{0}^{t} G(\varkappa, t, \tau, f(\varkappa, t)) d \tau\right\|
$$

Now, we introduce the following hypotheses:

(H1) There exists a nonnegative continuous function $g(\varkappa, t, \xi, \tau)$ defined on $D^{2}$ such that

$$
\left\|G\left(\varkappa, t, \xi, \tau, y_{1}\right)-G\left(\varkappa, t, \xi, \tau, y_{2}\right)\right\| \leq g(\varkappa, t, \xi, \tau)\left\|y_{1}-y_{2}\right\| .
$$

and

$$
\int_{0}^{t} \int_{\Omega} g(\varkappa, t, \xi, \tau) \exp (\mu(\xi+\|\tau\|)) \leq Q \exp (\mu(\varkappa+\|t\|)),
$$

where $\left(\varkappa, t, \xi, \tau, y_{i}\right) \in D^{2} \times \mathbb{R}^{n}, i=0,2$ and $Q \leq 0$.

(H2) There exists a constant $N>0$ such that

$$
f(\varkappa, t)+\int_{0}^{t} \int_{\Omega}\|G(\varkappa, t, \xi, \tau, 0)\| d \xi d \tau \leq N \exp (\mu(\varkappa+\|t\|)) .
$$

Theorem 4.8. Assumes that (H1) and (H2) hold, and if $0<Q<1$. Then there exists a unique solution of Eq.(1).

Proof. Let $\mathbb{S}$ is a space of all continuous functions $\phi: D \longrightarrow \mathbb{R}^{n}$ in $D$ satisfied

$$
\|\phi(\varkappa, t)\|=O \exp (\mu(\varkappa+\|t\|)),(\varkappa, t) \in D, \mu>0,
$$

then there exists a constant $M>0$ such that

$$
\|\phi(\varkappa, t)\|=M \exp (\mu(\varkappa+\|t\|)) .
$$

Thus, we get $|\phi| \leq M$.

It is easily seen that $\mathbb{S}$ with the norm $|\phi|=\sup _{D}[\|\phi(\varkappa, t)\| \exp (-\mu(\varkappa+\|t\|))]$ is a Banach space. 
Now, let the operator $T: \mathbb{S} \longrightarrow \mathbb{S}$ be defined by the right side of the equation (1). Evidently $T(y)$ is continuous in $D$ and $T(y(\varkappa, t)) \in \mathbb{R}^{n}$ for $y \in \mathbb{S}$ and $(\varkappa, \tau) \in D$.

Firstly, We prove that 48 is satisfactory, by assumptions (H1) and (H2) we have

$$
\begin{aligned}
T(y(\varkappa, t)) & \leq \int_{0}^{t} \int_{\Omega}\|G(\varkappa, t, \xi, \tau, y(\xi, \tau))-G(\varkappa, t, \xi, \tau, 0)\| d \xi d \tau \\
& +\|f(\varkappa, t)\|+\int_{0}^{t} \int_{\Omega} \| G(\varkappa, t, \xi, \tau, 0) d \xi d \tau \\
& \leq|y| \int_{0}^{t} \int_{\Omega} g(\varkappa, t, \xi, \tau) \exp (\mu(\xi+\|\tau\|)) d \xi d \tau+N \exp (\mu(\varkappa+\|t\|)) \\
& \leq[M Q+N] \exp (\mu(\xi+\|\tau\|)) .
\end{aligned}
$$

So, $T(y) \in \mathbb{S}$.

Secondly, we will prove that $T(y)$ is a contraction map. We assume that $y_{1}, y_{1} \in \mathbb{S}$, then from(H1) we have

$$
\begin{aligned}
\left\|T\left(y_{1}(\varkappa, t)\right)-T\left(y_{2}(\varkappa, t)\right)\right\| & \leq \int_{0}^{t} \int_{\Omega}\left\|G\left(\varkappa, t, \xi, \tau, y_{1}(\xi, \tau)\right)-G\left(\varkappa, t, \xi, \tau, y_{2}(\xi, \tau)\right)\right\| d \xi d \tau \\
& \leq\left|y_{1}-y_{2}\right| \int_{0}^{t} \int_{\Omega} g(\varkappa, t, \xi, \tau) \exp (\mu(\xi+\|\tau\|)) d \xi d \tau \\
& \leq Q\left|y_{1}-y_{2}\right| \exp (\mu(\xi+\|\tau\|)) .
\end{aligned}
$$

Consequently, we have

$$
\mid T\left(y_{1}-T\left(y_{2}|\leq Q| y_{1}-y_{2} \mid .\right.\right.
$$

As a result, $T$ is the contraction map. We can deduce from the Banach contraction principle that $T$ has a unique fixed point $y$ in $\mathbb{S}$.

\section{Numerical Results}

Numerical examples are investigated in this section by the proposed methods.

Example 1 : Consider the following NMVFIE

$$
y(\varkappa, t)=\varkappa t-e^{t}+t+1+\int_{0}^{t} \int_{0}^{1} t e^{y(\xi, \tau)} d \xi d \tau, \quad 0 \leq t \leq 1,
$$

which has the exact solutoin $y(\varkappa, t)=\varkappa t$

1. Using VIM to solve Eq. 49, we have this iteration formula

$$
y_{n+1}(\varkappa, t)=y_{n}(\varkappa, t)-\int_{0}^{t}\left[\frac{\partial y_{n}}{\partial \tau}(\varkappa, \tau)-\tau \int_{0}^{1} e^{y_{n}(\xi, \tau)} d \xi+e^{\tau}-\varkappa-1\right] d \tau,
$$

with the initial iteration $y_{0}(\varkappa, t)=0$. Using the iteration formula (50), the approximate solution convergents to the exact solution.

2. Using ADM and MADM. Subsitituting the series 201 to solve Eq. 49, we have

$$
\sum_{n=0}^{\infty} y_{n}(\varkappa, t)=\varkappa t-e^{t}+t+1+\int_{0}^{t} \int_{0}^{1} t\left(\sum_{n=0}^{\infty} A_{n}\right) d \xi d \tau
$$


where $A_{n}$ are the Adomian polinomial that calculating for nonlinear operator $H(y)=e^{y(\xi, \tau)}$ as follows

$$
\begin{aligned}
& A_{0}=H\left(y_{0}\right) \\
& A_{1}=y_{1} H^{\prime}\left(y_{0}\right) \\
& A_{2}=y_{2} H^{\prime}\left(y_{0}\right)+\frac{1}{2} y_{1}^{2} H^{\prime \prime}\left(y_{0}\right) \\
& \vdots
\end{aligned}
$$

Now, we decompose $f(\varkappa, t)$ into $f_{0}$ and $f_{1}$ as follows

$$
\begin{aligned}
& f_{0}=\varkappa t \\
& f_{1}=-e^{t}+t+1 .
\end{aligned}
$$

Applying MADM, we get

$$
\begin{aligned}
& y_{0}(\varkappa, t)=\varkappa t, \\
& y_{1}(\varkappa, t)=-e^{t}+t+1+\int_{0}^{t} \int_{0}^{1} t\left(A_{0}\right) d \xi d \tau, \\
& y_{k+2}(\varkappa, t)=\int_{0}^{t} \int_{0}^{1} t\left(A_{k+1}\right) d \xi d \tau, \quad k \geq 0,
\end{aligned}
$$

which implies

$$
\begin{aligned}
& y_{0}(\varkappa, t)=\varkappa t \\
& y_{1}(\varkappa, t)=-e^{t}+t+1+\int_{0}^{t} \int_{0}^{1} t\left(A_{0}\right) d \xi d \tau=0, \\
& y_{j}(\varkappa, t)=0, \quad j \geq 2 .
\end{aligned}
$$

Therefor $y(\varkappa, t)=\varkappa t$ which is convergenting to the exact soluation.

3. Using HPM. Same MADM, we decompose $f(\varkappa, t)$ into $f_{0}$ and $f_{1}$ as follows

$$
\begin{aligned}
& f_{0}=\varkappa t \\
& f_{1}=-e^{t}+t+1,
\end{aligned}
$$

To solve Eq. 49, we are using the recursive relation given as:

$$
\begin{aligned}
& p^{0}: y_{0}(\varkappa, t)=\varkappa t \\
& p^{1}: y_{1}(\varkappa, t)=-e^{t}+t+1+\int_{0}^{t} \int_{0}^{1} t e^{y(\xi, \tau)} d \xi d \tau \\
& \vdots
\end{aligned}
$$

which gives

$$
\begin{aligned}
& y_{0}(\varkappa, t)=\varkappa t \\
& y_{1}(\varkappa, t)=0, \\
& y_{j}(\varkappa, t)=0, \quad j \geq 2 .
\end{aligned}
$$

Therefor $y(\varkappa, t)=\varkappa t$ which is convergenting to the exact soluation. 
Table 1: Approximation solutions of Example 2.

\begin{tabular}{|c||c||c||c|}
\hline$(x, t)$ & $E_{2}(H P M) y_{1}$ & $E_{3}(V I M) y_{1}$ & $E_{n}(A D M) y_{1}$ \\
\hline$(0,0)$ & 0 & 0 & 0 \\
$(0.1,0.1)$ & $0.5630 \times 10^{-9}$ & $2.0 \times 10^{-13}$ & $8.7 \times 10^{-7}$ \\
$(0.2,0.2)$ & $0.7517 \times 10^{-7}$ & $1.1 \times 10^{-10}$ & $1.9 \times 10^{-5}$ \\
$(0.3,0.3)$ & $0.1371 \times 10^{-5}$ & $4.0 \times 10^{-9}$ & $8.8 \times 10^{-5}$ \\
$(0.4,0.4)$ & $0.1117 \times 10^{-4}$ & $7.5 \times 10^{-8}$ & $1.2 \times 10^{-4}$ \\
$(0.5,0.5)$ & $0.5881 \times 10^{-4}$ & $6.7 \times 10^{-7}$ & $3.7 \times 10^{-4}$ \\
$(0.6,0.6)$ & $0.2348 \times 10^{-3}$ & $4.2 \times 10^{-6}$ & $2.8 \times 10^{-3}$ \\
$(0.7,0.7)$ & $0.7739 \times 10^{-3}$ & $2.1 \times 10^{-5}$ & $1.0 \times 10^{-2}$ \\
$(0.8,0.8)$ & $0.2214 \times 10^{-2}$ & $8.8 \times 10^{-5}$ & $3.0 \times 10^{-2}$ \\
\hline
\end{tabular}

Example 2: Consider the following NSMVFIE

$$
\begin{aligned}
& y_{1}(\varkappa, t)=\frac{1}{6}\left(\varkappa^{2}+t^{2}\right)\left(t \cos (t)-\sin (t)-\frac{1}{2} \varkappa \sin (t)\right)+\int_{0}^{t} \int_{0}^{1}\left(\varkappa^{2}+t^{2}\right) \xi \tau y_{1}(\xi, \tau) d \xi d \tau, \\
& y_{2}(\varkappa, t)=0.14726 t^{3}(t-\varkappa)+t \tan (\varkappa)+\int_{0}^{t} \int_{0}^{1}\left(\xi(t-\varkappa) y_{2}^{2}(\xi, \tau)\right) d \xi d \tau,
\end{aligned}
$$

which posses the exact solutoins $y_{1}(\varkappa, t)=-\frac{\varkappa}{2} \sin (t), y_{2}(\varkappa, t)=t \tan (\varkappa)$.

Table 1 indicates the comparison between the errors are obtained by the HPM, VIM and ADM respectively for $y_{1}(\varkappa, t)$ and we can see that the errors of $y_{2}(\varkappa, t)$ are zeros because the approximate soluations and the exact solutions are equal. It is shown in Table 1 that the solution found by the proposed methods nearly congruous to the exact solution. In this example the precision and simplicity of the proposed methods are demonstrated by calculating the absolute error. The accuracy of the analysis can be increased by adding a more approximate solution. There is a strong concurrence between the exact solution and the approximate solution obtained using the proposed methods.

\section{Conclusion}

HPM, MHPM, ADM, MADM, VIM have been applied for solving a class of nonlinear problems effectively, easily and precisely with approximations that are converging rapidly to the exact solutions. In this paper, we have investigated the approximate solution of NMVFIEs and NSVFIE via the proposed methods. The proposed methods require much less calculation work compared to conventional methods and have been widely used to find an approximate solution for the analytical methods of the nonlinear mixed Volterra-Fredholm integral equation and its system. Also, the theoretical rseults such as the convergence and uniqueness of the solution of the suggested methods for the considered problems have been proved. Moreover, the validity and efficiency of these methods have been demonstrated through various numerical examples that illustrate the efficiency, accuracy, and simplicity of the proposed methods. We can see that variational iteration method is very effective and highly promising.

\section{Acknowledgments}

The authors would like to thank the editor and reviewers of journal of Results in Nonlinear Analysis for their careful reading of our manuscript and their helpful comments and suggestions. 


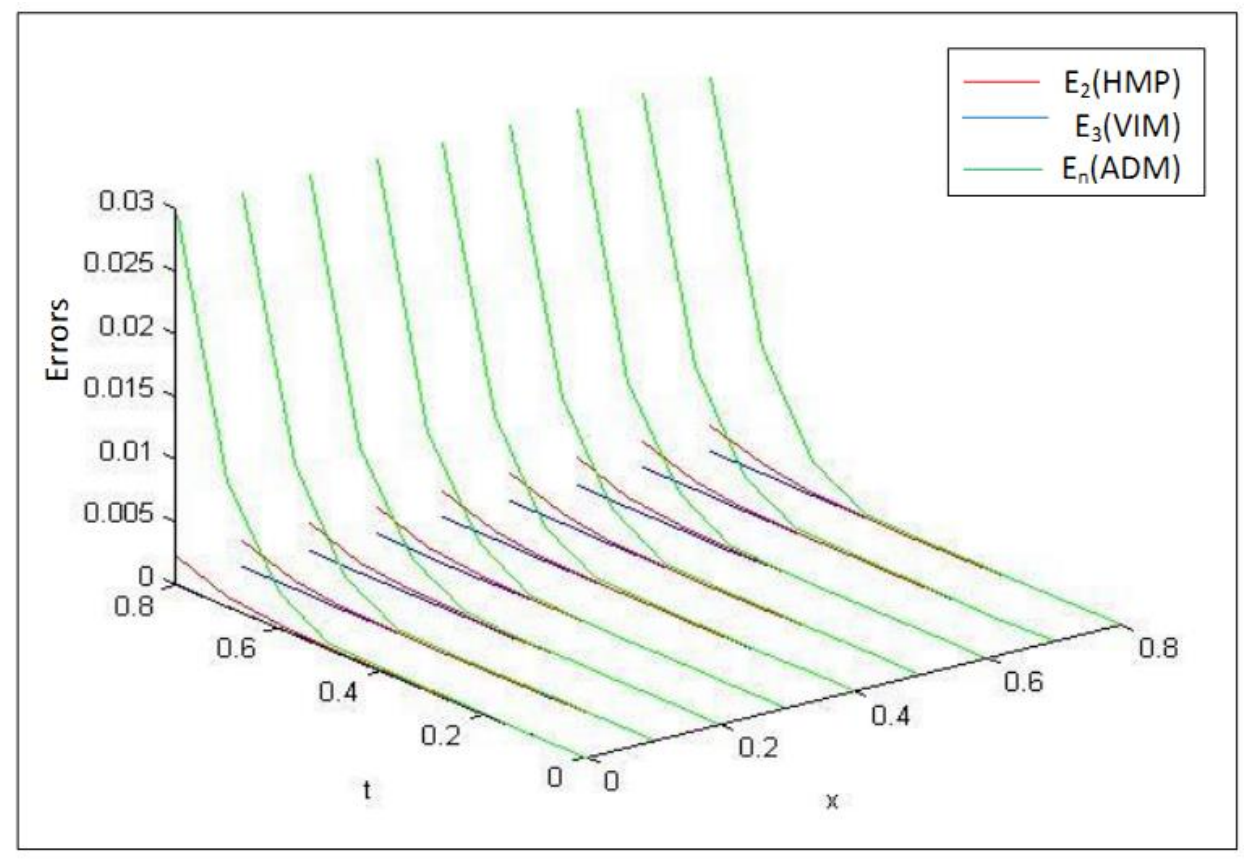

Figure 1: Absolute error for $y_{1}(\varkappa, t)$ in Example 2.

\section{References}

[1] M.S. Abdo, and S.K. Panchal, Some new uniqueness results of solutions to nonlinear fractional integro-differential equations. APAM, 2018, 16, 345-352.

[2] M.S. Abdo, A.M. Saeed, H.A. Wahash and S.K. Panchal, On nonlocal problems for fractional integro-differential equation in Banach space, Eur. J. Sci. Res. 2019, 151, 320-334.

[3] Q.M. Al-Mdallal, Monotone iterative sequences for nonlinear integro-differential equations of second order, Nonlinear Anal. Real World Appl. 2011, 12, 3665-3673.

[4] F.M. Al-Saar, K.P. Ghadle, and P.A. Pathade, The approximate solutions of Fredholm integral equations by Adomian decomposition method and its modification, Int. J. Math. Appl. 2018, 6, 327-336.

[5] F.M. Al-Saar and K.P. Ghadle, An approximate solution for solving the system of Fredholm integral equations of the second kind, Bull. Pure Appl. Sci. Math. 2019, 1, 208-215.

[6] M. Asif, I. Khan, N. Haider, Q. Al-Mdallal, Legendre multi-wavelets collocation method for numerical solution of linear and nonlinear integral equations, Alex. Eng. J. 2020, 59, 5099-5109.

[7] E. Babolian and J. Biazar, Solution of a system of linear Volterra equations by Adomian decomposition method, Far East J. Math. Sci. 2002, 2, 935-945.

[8] S.S. Behzadi, The use of iterative methods to solve two-dimensional nonlinear Volterra-Fredholm integro-differential equations, Commun. Math. Anal. 2012, 2012, 1-20.

[9] J. Biazar, B. Ghanbari, M. Porshokouhi and M. Porshokouhi, He's homotopy perturbation method: a strongly promising method for solving non-linear systems of the mixed Volterra-Fredholm integral equations, Comput. Math. with Appl. 2011, 61, 1016-1023.

[10] C. Dong, Z. Chen and W. Jiang, A modified homotopy perturbation method for solving the nonlinear mixed VolterraFredholm integral equation, J. Comput. Appl. Math. 2013, 239, 359-366.

[11] A.A. Hamoud and K.P. Ghadle, Modified Adomian decomposition method for solving fuzzy Volterra-Fredholm integral equations, J. Indian Math. Soc. 2018, 85, 53-69.

[12] A.A. Hamoud and K.P. Ghadle, Recent advances on reliable methods for solving Volterra-Fredholm integral and integrodifferential equations, Asian J. Math. Comput. Res. (2018), 24, 128-157.

[13] A.A. Hamoud and K.P. Ghadle, The combined modified Laplace with Adomian decomposition method for solving the nonlinear Volterra-Fredholm integro-differential equations, J. Korean Soc. Ind. Appl. Math. (2017),21, 17-28.

[14] A.A. Hamoud and K.P. Ghadle, The approximate solutions of fractional Volterra-Fredholm integro-differential equations by using analytical techniques, Probl. Anal. Issues Anal. (2018),25, 41-58.

[15] J.H. He, Homotopy perturbation technique, Comput. Methods Appl. Mech. Engrg. (1999),178, 257-262.

[16] F.A. Hendi, and M.M. Al-Qarni, Numerical treatment of nonlinear Volterra-Fredholm integral equation with a generalized singular kernel, Am. J. Comput. Math. (2016), 6, 245-250.

[17] F.A. Hendia and M.M. Al-Qarnib, Numerical solution of nonlinear mixed integral equations with singular Volterra kernel, Int. J. Adv. Appl. Math. and Mech. 2016, 3, 41-48. 
[18] I. Khan, M. Asif, Q. Al-Mdallal, F. Jarad, On a new method for finding numerical solutions to integro-differential equations based on Legendre multi-wavelets collocation, Alex. Eng. J. 2021, 1-13.

[19] K. Maleknejad, K. Nouri and L. Torkzadeh, Comparison projection method with Adomian's decomposition method for solving system of integral equations, Bull. Malays. Math. Sci. Soc. (2011),34, 379-388.

[20] F. Mirzaee and A.A. Hoseini, Numerical solution of nonlinear Volterra-Fredholm integral equations using hybrid of blockpulse functions and Taylor series, Alex. Eng. J. 2013, 52, 551-555.

[21] M.H. Saleh, D.S. Mohamed, and R.A. Taher, Variational Iteration Method for Solving Two Dimensional Volterra-Fredholm Nonlinear Integral Equations, Int. J. Comput. Appl. 2016, 152, 29-33.

[22] N.A. Sulaiman, Some numerical methods to solve a system of Fredholm integral equations of the 2nd kind with symmetric kernel, Kirkuk Univ. J. Sci. Stud. (2009), 4, 108-116.

[23] R.K. Pandey, O.P. Singh and V.K. Singh, Efficient algorithms to solve singular integral equations of Abel type, Comput. Math. with Appl. (2009), 57, 664-676.

[24] M. Rabbani and R. Jamali, Solving nonlinear system of mixed Volterra-Fredholm integral equations by using variational iteration method, J. Math. Comput. Sci. 2012, 5, 280-287.

[25] H.A. Wahash, M.S. Abdo and S.K. Panchal, An existence result for fractional integro-differential equations on Banach space, J. Math. Ext. 2019, 13, 19-33.

[26] H.A. Wahash, M.S. Abdo, A.M. Saeed and S.K. Panchal, Singular fractional differential equations with $\psi$-Caputo operator and modified Picard's iterative method, Appl. Math. E-Notes, 2020, 20, 215-229.

[27] A.M. Wazwaz, A reliable treatment for mixed Volterra-Fredholm integral equations, Appl. Math. and Comput. 2002, 127, 405-414.

[28] S.A. Yousefi, A. Lotfi and M. Dehghan, He's variational iteration method for solving nonlinear mixed Volterra-Fredholm integral equations, Comput. Math. with Appl. 2009, 58, 2172-2176. 